\title{
Treatment of chronic phantom limb pain using a trauma-focused psychological approach
}

\author{
C de Roos MA, AC Veenstra MA, Prof A de Jongh PhD, ME den Hollander-Gijsman MA, \\ NJA van der Wee PhD, Prof FG Zitman PhD, YR van Rood PhD
}

\begin{abstract}
C de Roos, AC Veenstra, A de Jongh, et al. Treatment of chronic phantom limb pain using a trauma-focused psychological approach. Pain Res Manage 2010;15(2):65-71.
\end{abstract}

BACKGROUND: Chronic phantom limb pain (PLP) is a disabling chronic pain syndrome for which regular pain treatment is seldom effective. Pain memories resulting from long-lasting preamputation pain or pain flashbacks, which are part of a traumatic memory, are reported to be powerful elicitors of PLP.

OBJECTIVE: To investigate whether a psychological treatment directed at processing the emotional and somatosensory memories associated with amputation reduces PLP.

METHODS: Ten consecutive participants (six men and four women) with chronic PLP after leg amputation were treated with eye movement desensitization and reprocessing (EMDR). Pain intensity was assessed during a two-week period before and after treatment (mean number of sessions $=5.9$ ), and at short- (three months) and long-term (mean 2.8 years) follow-up.

RESULTS: Multivariate ANOVA for repeated measures revealed an overall time effect $(\mathrm{F}[2,8]=6.7 ; \mathrm{P}<0.02)$ for pain intensity. Pairwise comparison showed a significant decrease in mean pain score before and after treatment $(\mathrm{P}=0.00)$, which was maintained three months later. All but two participants improved and four were considered to be completely pain free at three months follow-up. Of the six participants available at long-term follow-up (mean 2.8 years), three were pain free and two had reduced pain intensity.

CONCLUSIONS: These preliminary results suggest that, following a psychological intervention focused on trauma or pain-related memories, substantial long-term reduction of chronic PLP can be achieved. However, larger outcome studies are required.

Key Words: Cortical reorganization; EMDR; Longitudinal descriptive study; Pain memories; Phantom limb pain (PLP); Post-traumatic stress disorder (PTSD)

$\mathrm{P}$ hantom limb pain (PLP) occurs in $50 \%$ to $80 \%$ of patients after an amputation $(1,2)$, and often leads to significant impairment in occupational and social functioning (3). Daily pain is reported by $48 \%$ of patients with PLP (4) and reviews have concluded that regular pain treatment is seldom effective $(5,6)$.

Both peripheral and central factors have been proposed as determinants of PLP. However, peripheral factors alone cannot be the primary or sole factor in PLP because it occurs even when no pathological features are present in the residual limb (7). Central factors - ie, cortical reorganization and the development of somatosensory memories (8-10) - have been identified that are strongly associated with the intensity of PLP. Two types of somatosensory memories are described - those

\section{Le traitement de la douleur chronique d'un membre fantôme, axé sur une approche psychologique}

HISTORIQUE : La douleur chronique d'un membre fantôme (DMF) est un syndrome de douleur chronique invalidant qui réagit rarement au traitement analgésique habituel. Il est établi que les souvenirs de la douleur, qui entrainent la présence permanente des douleurs précédant l'amputation ou des rappels de la douleur partiellement attribuables à un souvenir traumatique, sont de puissants déclencheurs de DMF.

OBJECTIF : Explorer si un traitement psychologique orienté vers l'analyse des souvenirs affectifs et somatosensoriels associés à l'amputation réduit la DMF.

MÉTHODOLOGIE : Dix participants consécutifs (six hommes et quatre femmes) ayant des DMF après l'amputation d'une jambe ont reçu un traitement par désensibilisation et retraitement par les mouvements oculaires. Les chercheurs ont évalué l'intensité de la douleur pendant une période de deux semaines avant et après le traitement (nombre moyen de séances $=5,9$ ) et dans le cadre d'un suivi à court (trois mois) et à long terme (moyenne de 2,8 ans).

RÉSULTATS : L'analyse de variance multivariée des mesures répétées a révélé un effet temporel global $(\mathrm{F}[2,8]=6,7 ; \mathrm{P}<0,02)$ d'intensité de la douleur. La comparaison par paire a révélé une diminution significative de l'indice de douleur moyen avant et après le traitement $(\mathrm{P}=0,00)$, maintenue trois mois plus tard. L'état de tous les participants, sauf deux, s'est amélioré, et on estimait que quatre participants ne ressentaient plus aucune douleur au suivi de trois mois. Des six participants disponibles au suivi à long terme (d'une moyenne de 2,8 ans), trois ne ressentaient plus de douleurs et deux profitaient d'une réduction de l'intensité de la douleur.

CONCLUSIONS : Selon ces résultats préliminaires, après une intervention psychologique axée sur les souvenirs des traumatismes ou de la douleur, on peut obtenir d'importantes réductions de la DMF chronique à long terme. Cependant, il faudra mener des études d'issue de plus grande envergure sur le sujet.

resulting from long-lasting preamputation pain, and pain flashbacks, which are part of a traumatic memory (9,11-13).

Katz and Melzack (9) proposed that a neural representation of the sensory qualities of the preamputation pain is formed when the patient is exposed to mild pain of long duration or intense pain of short duration. Once this neural network has been formed, it can be activated even when only some of its elements are present in the sensory input (14). The precise mechanisms are not yet known, but both the loss of normal afferent input following amputation and the associated process of cortical reorganization seem to play a role $(7,15)$.

Pain flashbacks have been described as a symptom of posttraumatic stress disorder (PTSD) $(11,12)$, but can also occur in subjects who no longer meet the criteria for PTSD (13). These

Mental Health Center 'Rivierduinen', Leiden, The Netherlands

Correspondence: Carlijn de Roos, Mental Health Center 'Rivierduinen', PO Box 2211, 2301 CE Leiden, The Netherlands.

Telephone 31-71-8908400, fax 31-71-8908401, e-mail c.deroos@ggzkinderenenjeugd.nl 
TABLE 1

Demographic and clinical characteristics of the 10 patients with phantom limb pain

\begin{tabular}{|c|c|c|c|c|c|c|c|c|c|c|}
\hline Patient & Sex & $\begin{array}{l}\text { Age, } \\
\text { years }\end{array}$ & $\begin{array}{l}\text { Reason for } \\
\text { amputation }\end{array}$ & $\begin{array}{l}\text { Amputation } \\
\text { side }\end{array}$ & Dominance & $\begin{array}{l}\text { Amputation } \\
\text { site }\end{array}$ & $\begin{array}{l}\text { Time since } \\
\text { amputation, } \\
\text { years }\end{array}$ & $\begin{array}{l}\text { Duration (months) } \\
\text { and type of } \\
\text { preamputation pain }\end{array}$ & $\begin{array}{l}\text { Type of } \\
\text { postamputation } \\
\text { pain }\end{array}$ & Medication \\
\hline 1 & $M$ & 51 & Accident & $\mathrm{L}$ and $\mathrm{R}$ & $\mathrm{R}$ & Above knee & 8.7 & 0 , pain free & Cramping, stabbing & Antiepileptic \\
\hline 2 & $\mathrm{~F}$ & 45 & $\begin{array}{l}\text { Accident and } \\
\text { medical failure }\end{array}$ & $\mathrm{L}$ & $\mathrm{R}$ & Above knee & 3.4 & 8 , burning & Stinging, stabbing & Alcohol \\
\hline 3 & $\mathrm{~F}$ & 45 & $\begin{array}{l}\text { Vascular disease } \\
\text { and medical } \\
\text { failure }\end{array}$ & $\mathrm{R}$ & $\mathrm{R}$ & Below knee & 1.6 & $>12$, cramping & Stabbing & $\begin{array}{l}\text { Antiepileptic, } \\
\text { antidepressant }\end{array}$ \\
\hline 4 & M & 59 & Accident & $\mathrm{L}$ & $\mathrm{R}$ & Upper leg & 35.8 & 0 , pain free & $\begin{array}{l}\text { Stabbing, stinging, } \\
\text { shooting }\end{array}$ & - \\
\hline 5 & $\mathrm{~F}$ & 54 & Medical failure & $\mathrm{L}$ & L (R-handed) & Below knee & 38.9 & $<6$, stinging, stabbing & Stabbing, shooting & - \\
\hline 6 & M & 33 & Accident & $\mathrm{R}$ & $\mathrm{R}$ & Pelvis & 13.1 & 0 , pain free & Stabbing & $\begin{array}{l}\text { Antiepileptic, } \\
\text { beta-blocker }\end{array}$ \\
\hline 7 & M & 64 & Cancer & $\mathrm{L}$ & $\mathrm{L}$ and $\mathrm{R}$ & Pelvis & 1.0 & $<6$, squeezing & $\begin{array}{l}\text { Cramping, stinging, } \\
\text { shooting }\end{array}$ & Antiepileptic \\
\hline 8 & M & 67 & Medical failure & $\mathrm{R}$ & $\mathrm{L}$ & Below knee & 1.0 & 0 , pain free & Stabbing & - \\
\hline 9 & M & 51 & Cancer & $\mathrm{L}$ & $\mathrm{L}$ & Pelvis & 28.4 & $>12$, aching & Stabbing, stinging & - \\
\hline 10 & $\mathrm{~F}$ & 32 & $\begin{array}{c}\text { Complex regional } \\
\text { pain syndrome }\end{array}$ & $\mathrm{R}$ & $\mathrm{R}$ & Upper leg & 3.8 & $\begin{array}{l}>12, \text { cramping, } \\
\text { burning }\end{array}$ & Cramping, burning & Opiate \\
\hline
\end{tabular}

F Female; L Left; $M$ Male; R Right

flashbacks resemble the pain experienced during the traumatic event and are triggered by specific environmental or internal stimuli, suggesting a classically conditioned response. The similarity of the flashbacks in quality and location to the original pain experience, the triggering of flashbacks by trauma-related cues, and the associated emotional arousal and avoidance suggest the involvement of a somatosensory memory mechanism (11).

Painful conditions, such as PLP, may be the result of inappropriately stored or chronically activated pain memories that continue to disturb the subject even after the disease or injury has been successfully treated $(10,13,16,17)$. These memories are a major factor in the maintenance of PLP, and disengagement of such memories may have a lasting effect on PLP. Methods that are potent in processing traumatic memories might also be effective in reducing the affective dimensions of pain memories to a situation-appropriate level (16).

An intervention specifically aimed at processing unresolved memories of negative experiences is eye movement desensitization and reprocessing (EMDR), an evidence-based treatment for traumatically induced memories $(18,19)$. Small case series $(17,20-23)$ have indicated that EMDR is effective in the treatment of PLP. However, limitations of these studies are that the presence of PTSD was not always assessed, no longterm follow-up data were collected, and pain intensity was based on the patient's current pain rather than assessing pain intensity over a longer period $(17,20,22,23)$.

The present study tested the effectiveness of a traumafocused psychological approach in the treatment of chronic PLP, using a standardized EMDR pain protocol. Mean pain intensity as the primary outcome measure was assessed over a two-week period, and a long-term follow-up was conducted.

\section{METHODS}

\section{Participants}

The present study included 10 consecutive participants (six men and four women) 32 to 67 years of age (mean [ \pm SD] age $50.1 \pm 10.1$ years) with chronic PLP after leg amputation (Table 1). Inclusion criteria were a history of at least 12 months PLP and severe disabling pain for at least five days per week. Participants were excluded from the study if a psychiatric disorder was diagnosed for which immediate treatment was necessary (eg, psychosis, dissociative disorder or risk of suicide). Medical exclusion criteria (related to possible complications during the EMDR process) were epilepsy and pregnancy. Participants were asked not to change their medication for PLP during the study period, and to refrain from using any other treatment for PLP for the duration of the study.

Reasons for amputation were accidents $(n=3)$, cancer $(n=2)$, medical failures $(n=2)$ and complex regional pain syndrome (CRPS) $(n=1)$. Two participants had their leg amputated due to a combination of factors. One patient was suffering from an undiagnosed vascular disease and, as a result, lost her leg, and the other participant lost her leg because a plaster cast was placed too tightly around her leg after a serious accident. Time since amputation ranged from one to 39 years (mean $13.6 \pm 15.1$ years).

Five participants had undergone leg amputation on the left side, and four on the right side. One participant had both legs amputated. The locations of amputation included below the knee $(n=3)$, above the knee $(n=2)$, upper leg $(n=2)$ and pelvis $(n=3)$. Four participants had no pain before amputation; in the remaining participants, the duration of preamputation pain was categorized as six months or less $(n=2)$, six to 12 months $(n=1)$ and one year or more $(n=3)$. Phantom pain was described as aching, squeezing, cramping, shooting, burning, stinging and stabbing. Four participants did not use any medication for PLP, whereas the others used beta-blockers, opiates, antiepileptics or antidepressant medication. One participant used alcohol to reduce the level of pain (Table 1).

The study was conducted at the Mental Health Center 'Rivierduinen' (Leiden, The Netherlands) and at the outpatient clinic of the 'St Elisabeth Hospital' (Tilburg, The Netherlands). Participants were recruited between September 
2003 and November 2004 from the departments of anesthesiology of Leiden University Medical Center (Leiden) and St Elisabeth Hospital, and through an announcement in the newsletter of the Dutch association of limb prosthesis users. All participants provided written informed consent. The local ethics committee stated that their approval was not required for the present pilot study.

\section{Experimental design and procedure}

The present study used a pretest/post-test design in which data were collected two weeks before and after treatment, at three months follow-up, and at long-term follow-up. The first contact with participants was by telephone. After obtaining informed consent, the participant's medical and psychiatric history was taken at the outpatient clinic. To explore the presence of axis I disorders according to the Diagnostic and Statistical Manual of Mental Disorders, Fourth Edition, Text Revision (DSM-IV-TR) diagnostic criteria, a short clinical diagnostic interview was used (Mini International Neuropsychiatric Interview Plus 5.0.0.-R) (24). After inclusion, all participants were assessed. The clinical diagnostic interview and questionnaires were administered and scored by research nurses from Rivierduinen and St Elisabeth Hospital. Treatment began two to four weeks after the assessment. The number of treatment sessions was not preset, but rigorous criteria were described for ending treatment.

\section{Primary outcome measure: Pain intensity}

Pain intensity was the primary outcome measure. All participants completed a symptom diary (for a two-week period), rating their pain intensity three times a day using a 0 to 10 numeric rating scale, in which 0 was defined as 'no pain at all' and 10 as the 'worst pain possible'. This was performed during the two weeks before treatment, following treatment, at three months follow-up and at long-term follow-up.

\section{Secondary outcome measures}

The Dutch version of the Symptom Checklist 90 (SCL-90) was used to measure psychological distress. The SCL-90 consists of eight subscales (somatic complaints, cognitive performance deficits, interpersonal sensitivity and mistrust, depression, anxiety, hostility, agoraphobia, and sleeping difficulties) and a total score (psychoneuroticism). Total scores range from 90 to 450 (25).

The Checklist Individual Strength - Revised (CIS-20R) was used to measure fatigue (26). The CIS-20R consists of four subscales (subjective tiredness, concentration, motivation and physical activity) and a total score ranging from 20 to 140 .

To assess intensity of PTSD symptoms, the Dutch version of the Impact of Events Scale (27) and the Self-Inventory List (SIL) (28) were used. The Impact of Events Scale consists of 15 items scored on a four-point scale. It has two subscales (avoidance and intrusions) and a total score ranging from 15 to 60 . The SIL consists of 22 items scored on a fourpoint scale. It has three subscales (avoidance, intrusions and hyperarousal) and a total score ranging from 22 to 88 .

Finally, the Dutch version of the Short Form-36 Health Survey (SF-36) (29) was used to measure generic health-related quality of life. The SF-36 consists of eight domains (physical functioning, role limitations due to physical and emotional problems, social functioning, bodily pain, mental health, vitality, and general health perception) with an outcome score of 0 to 100 . Higher scores indicate a better quality of life.

\section{Treatment}

Shapiro (19) describes EMDR as a structured, multicomponent treatment method that incorporates the following eight stages:

1. Recording a medical history and planning treatment.

2. Explanation of and preparation for EMDR.

3. Assessment: Identification of a distressing image in memory (target) and associated negative cognition, affect and body sensations. In addition, an alternative positive cognition is selected and the validity of cognition is measured using a scale from 1 ('completely false') to 7 ('completely true'). The level (or unit) of subjective disturbance is also measured, using a scale from 0 ('no disturbance') to 10 ('extreme disturbance').

4. Desensitization of the target: The therapist asks the patient to keep the disturbing target image and related aspects in mind, while simultaneously focusing on the bilateral stimulation introduced by the therapist. Bilateral stimulation consists of alternating left-to-right eye movements, ear tones or hand tapping, at a rate of approximately two movements, tones or taps per second for approximately $45 \mathrm{~s}$. After this 'set', the patient briefly reports what has come to mind. This procedure is repeated until the initial target memory is no longer disturbing.

5. Installation of the positive cognition: The patient is instructed to think of the target image and to rehearse the positive cognition while bilateral stimulation is added until it feels completely true.

6. Body scan: Check for any signs of residual physical tension or discomfort. If any of these signs are reported, the patient is instructed to focus on the physical sensations, while bilateral stimulation is added until the tension is reduced or has disappeared.

7. Closure of the session: Preparation of the patient to leave the session.

8. Re-evaluation in the following session: The patient comments on previously processed targets and, if necessary, a new target is selected for the next session.

The number of EMDR sessions is determined by the number of traumatic memories (targets) to be accessed and resolved.

In the present study, in addition to trauma targets, painrelated targets and in-session experiences (targets) of PLP were selected for treatment. These three types of EMDR memories were targeted and processed one after another in the abovementioned order. Trauma targets consisted of actual disturbing memories of traumatic events (eg, the accident leading to the amputation), as well as the events following amputation. Next, pain-related disturbing memories were targeted. These were actual disturbing memories related to the consequences of the loss of a limb and resulting physical disability, or memories underlying inadequate coping with the pain (eg, an upsetting and painful memory of falling on the stump one year earlier, after which the patient still feels powerless). Finally, EMDR was directed at actual PLP as experienced during the session. The standard EMDR protocol was used for the first and second 
type of targets (19). For processing of in-session PLP, an EMDR pain protocol (30) was developed that combined the standard EMDR protocol (19) and the pain protocol of Grant (31). The latter protocol differs from the standard trauma protocol in that it focuses on currently experienced PLP and phantom limb sensations instead of the traumatic memory. In the present study, eye movements or auditory tones were used for bilateral stimulation. Because both methods were assumed to be effective $(19,32)$, it was left to the therapist and/or patient to decide which one to use. EMDR treatment consisted of weekly $90 \mathrm{~min}$ sessions and was conducted shortly after recruitment in the study. Two senior psychotherapists trained in EMDR ( $\mathrm{C}$ de $\mathrm{R}$ and ACV) delivered treatment. Treatment ended when the participant reported no more pain at the end of the session, or reported no further reduction in the level of disturbance related to trauma and pain-related targets, or in the level of pain intensity (actual pain target) during three successive sessions. In the present study, the mean number of sessions was 5.9 (range three to 10$)$.

\section{Data analysis}

Data were analyzed on an intention-to-treat basis with the last observation carried forward. Participant 9 resigned from the study after the fourth EMDR session.

Whenever one or more (but not all) items of a subscale were missing, the mean score of the remaining items of the same subscale replaced the missing item values for that individual. In total, five missing values were replaced.

To detect differences between our sample and the general population, the one-sample $t$ test was used. $\mathrm{P} \leq 0.05$ was considered to be significant. To determine whether the scores on the primary outcome measure (pain diary) changed over time, a general linear model repeated measures analysis was performed. When the general linear model revealed a significant $(\mathrm{P} \leq 0.05)$ time effect, the contrast test was interpreted $($ eg, type $=$ simple $)$ and the mean of each level was compared with the mean of level 1 (pretest). Effect sizes were calculated as eta-squared.

All statistical analyses were performed using SPSS 11.0 for Windows (SPSS Inc, USA).

\section{Participants' characteristics}

\section{RESULTS}

The results of the clinical diagnostic interview (Mini International Neuropsychiatric Interview Plus 5.0.0.-R) showed that all participants (except for one) had a normal profile. Participant 2 fulfilled DSM-IV-TR criteria for PTSD, obsessive-compulsive disorder, alcohol dependency and adjustment disorder with depressed mood. This participant used alcohol to reduce the level of pain. All participants fulfilled the diagnostic criteria for pain disorder.

Compared with the Dutch general population, the present sample had more symptoms of hyperarousal (SIL), more sleeping difficulties (SCL-90), felt more tired, had more problems concentrating and had a higher total score on the CIS-20R. Furthermore, physical and social functioning were more impaired, and they reported more bodily pain (SF-36) than the reference group (Table 2).

\section{Primary outcome measure: Pain intensity}

The mean pain scores of the pain diary were $5.0 \pm 1.7$ at pretest, $2.8 \pm 2.6$ at post-test and $2.5 \pm 2.9$ at follow-up. There was an overall time effect $(\mathrm{F}[2,8]=6.7 ; \mathrm{P}=0.02)$, which corresponds with an average effect size (eta-squared $=0.60$ ). Pairwise comparison showed a significant decrease in mean pain scores before and after treatment $(\mathrm{P}=0.00)$, which was maintained at follow-up. Two participants (9 and 10) did not improve. Four participants $(2,4,7$ and 8$)$ reported a clinically relevant reduction in pain intensity (range $32 \%$ to $57 \%$ ) but were not pain free at follow-up, whereas four other participants $(1,3,5$ and 6$)$ were considered to be pain free (score less than 1) at follow-up (Figure 1). Those who were pain free stopped using medication.

\section{Secondary outcome measures}

As shown in Table 2, all mean scores decreased or remained stable over time. A significant change over time, which was maintained at follow-up, was observed for the interpersonal sensitivity and mistrust subscale and the total score of the SCL-90, as well as for the vitality and bodily pain subscales of the SF-36.

With the exception of the concentration subscale of the CIS-20R and the physical functioning subscale of the SF-36, all scores that differed from the reference group at pretest normalized at follow-up. This was even the case for the bodily pain subscale of the SF-36.

\section{Side effects during treatment}

All participants felt very tired after the sessions and four of them reported headaches during the session. Three participants complained of an increase in PLP during or immediately following the EMDR sessions, especially after sessions in which actual pain and/or pain-related memories were targeted. Pain continued for several hours after the session, but in all cases disappeared after one night's sleep. No other side effects were mentioned.

\section{Long-term follow-up}

To collect data for long-term follow-up, all participants were approached again (by telephone) in April 2007 and were sent a booklet containing the pain diary with the request to rate their pain intensity over 14 days, three times a day. At that time, the mean duration since treatment was 2.8 years (range 26 to 40 months). Of the nine participants approached (participant 9, a nonresponder, had resigned from the study during treatment), six responded (participants 1, 2, 3, 4, 5 and 10); participant 7 (who had shown improvement) had died from cancer, and participant 6 (who was pain free at three months follow-up) fell on his stump just before the assessment. This fall substantially increased his PLP because of peripheral nerve damage to the stump. Finally, participant 8 stated that he had insufficient time to fill in the diary due to administrative overload and ongoing legal procedures; he reported by telephone that his intensity of pain had not changed since the last assessment (three months after treatment). From the six available subjects at long-term follow-up, the pain intensity of two participants ( 3 and 4$)$ had decreased further (mean pain intensity 0.19 and 0.71 , respectively) since completion of treatment, and both subjects could now be considered pain free. The pain intensity of two other participants (2 and 5) had remained at the same level (mean pain intensity 1.67 and 0.20 , respectively). The pain intensity for participant 1 had increased (mean pain intensity 2.93), but was still substantially lower than at the start of treatment. Only participant 10 had no 
TABLE 2

Results of repeated measures on primary and secondary outcome measures

\begin{tabular}{|c|c|c|c|c|c|c|c|c|}
\hline \multirow[b]{2}{*}{ Measure } & \multirow[b]{2}{*}{ Subscale } & \multirow{2}{*}{$\begin{array}{l}\text { Normative } \\
\text { data, mean }\end{array}$} & \multirow[b]{2}{*}{ Pretest } & \multirow[b]{2}{*}{ Post-test } & \multirow[b]{2}{*}{ Follow-up } & \multicolumn{3}{|c|}{ Time effect } \\
\hline & & & & & & $F(2,8)$ & $\mathbf{P}$ & $\eta^{2}$ \\
\hline Pain diary & Pain & - & $5.0 \pm 1.7$ & $2.8 \pm 2.6$ & $2.5 \pm 2.9^{\star}$ & 6.7 & 0.02 & 0.63 \\
\hline \multirow[t]{9}{*}{ SCL-90 } & Somatization & 16.6 & $18.0 \pm 3.7$ & $15.6 \pm 4.6$ & $15.7 \pm 3.8$ & 3.3 & 0.06 & 0.27 \\
\hline & Cognitive performance deficits & 13.2 & $16.4 \pm 5.9$ & $12.2 \pm 2.3$ & $13.1 \pm 4.1$ & 5.7 & 0.01 & 0.39 \\
\hline & Interpersonal sensitivity and mistrust & 24.6 & $24.3 \pm 6.3$ & $19.5 \pm 1.8$ & $20.3 \pm 3.6^{*}$ & 7.2 & 0.02 & 0.45 \\
\hline & Depression & 20.7 & $23.4 \pm 7.2$ & $18.4 \pm 2.4$ & $19.4 \pm 3.9$ & 4.4 & 0.06 & 0.33 \\
\hline & Anxiety & 13.0 & $12.9 \pm 3.0$ & $11.3 \pm 1.2$ & $11.4 \pm 1.9$ & 3.1 & 0.07 & 0.26 \\
\hline & Acting-out hostility & 7.5 & $7.6 \pm 2.1$ & $6.6 \pm 0.5$ & $7.0 \pm 1.1$ & 1.9 & 0.19 & 0.19 \\
\hline & Agoraphobia & 7.9 & $8.3 \pm 1.8$ & $7.7 \pm 1.1$ & $7.3 \pm 0.7$ & 2.4 & 0.15 & 0.21 \\
\hline & Sleeping difficulties & 4.6 & $7.5 \pm 4.0^{\dagger}$ & $5.2 \pm 2.5$ & $5.8 \pm 2.7^{\ddagger}$ & 3.8 & 0.04 & 0.30 \\
\hline & Total & 117.2 & $129.0 \pm 26.4$ & $106.4 \pm 8.7$ & $109.8 \pm 16.3^{\star}$ & 8.6 & 0.01 & 0.49 \\
\hline \multirow[t]{5}{*}{ CIS-20R } & Subjective tiredness & 17.3 & $31.6 \pm 13.0^{\dagger}$ & $22.7 \pm 13.6$ & $25.6 \pm 16.3^{\ddagger}$ & 4.3 & 0.03 & 0.32 \\
\hline & Concentration & 6.6 & $17.4 \pm 7.4^{\dagger}$ & $11.9 \pm 7.1$ & $14.9 \pm 8.9$ & 4.8 & 0.02 & 0.35 \\
\hline & Motivation & 7.9 & $11.0 \pm 5.1$ & $7.4 \pm 2.0$ & $7.9 \pm 3.8$ & 3.3 & 0.06 & 0.27 \\
\hline & Physical activity & 9.5 & $8.6 \pm 3.7$ & $6.5 \pm 2.8$ & $6.4 \pm 3.1$ & 6.8 & 0.01 & 0.43 \\
\hline & Total & 41.5 & $68.8 \pm 18.9^{\dagger}$ & $48.5 \pm 21.1$ & $54.8 \pm 24.6^{\ddagger}$ & 7.5 & 0.04 & 0.45 \\
\hline \multirow[t]{3}{*}{ IES } & Intrusions & 6.6 & $8.7 \pm 8.0$ & $2.3 \pm 2.8$ & $5.3 \pm 6.5$ & 6.3 & 0.01 & 0.41 \\
\hline & Avoidance & 6.9 & $6.8 \pm 10.9$ & $1.1 \pm 1.9$ & $2.9 \pm 5.9$ & 2.6 & 0.10 & 0.23 \\
\hline & Total & 13.3 & $15.9 \pm 17.9$ & $3.5 \pm 3.9$ & $8.2 \pm 11.4$ & 5.1 & 0.02 & 0.36 \\
\hline \multirow[t]{4}{*}{ SIL } & Intrusions & 7.1 & $7.6 \pm 2.2$ & $6.7 \pm 1.6$ & $6.7 \pm 1.6$ & 4.3 & 0.06 & 0.33 \\
\hline & Avoidance & 11.9 & $11.9 \pm 2.8$ & $10.5 \pm 2.0$ & $11.2 \pm 3.2$ & 1.3 & 0.29 & 0.13 \\
\hline & Hyperarousal & 10.1 & $12.3 \pm 2.3^{\dagger}$ & $10.0 \pm 1.8$ & $11.5 \pm 3.0^{\ddagger}$ & 3.8 & 0.04 & 0.30 \\
\hline & Total & 29.0 & $31.8 \pm 5.3$ & $27.2 \pm 4.6$ & $29.4 \pm 5.7$ & 5.9 & 0.01 & 0.40 \\
\hline \multirow[t]{8}{*}{ SF-36§ } & Physical functioning & 83.0 & $29.5 \pm 15.2^{\dagger}$ & $42.0 \pm 30.7$ & $35.0 \pm 22.2$ & 1.1 & 0.36 & 0.11 \\
\hline & Social functioning & 84.0 & $71.3 \pm 15.6^{\dagger}$ & $83.8 \pm 15.6$ & $88.8 \pm 15.0^{\ddagger}$ & 4.8 & 0.02 & 0.35 \\
\hline & Role physical & 76.4 & $52.5 \pm 44.8$ & $60.0 \pm 51.6$ & $62.5 \pm 42.9$ & 0.3 & 0.63 & 0.04 \\
\hline & Role emotional & 82.3 & $90.0 \pm 22.5$ & $93.3 \pm 21.1$ & $90.0 \pm 31.6$ & 0.1 & 0.84 & 0.01 \\
\hline & Mental health & 76.8 & $80.4 \pm 9.5$ & $82.4 \pm 7.6$ & $80.0 \pm 12.6$ & 0.4 & 0.70 & 0.04 \\
\hline & Vitality & 68.8 & $58.5 \pm 14.9$ & $71.0 \pm 14.3$ & $68.0 \pm 19.5^{\star}$ & 6.7 & 0.01 & 0.43 \\
\hline & Bodily pain & 74.9 & $45.5 \pm 20.1^{\dagger}$ & $63.5 \pm 23.5$ & $67.8 \pm 27.7^{\star \ddagger}$ & 8.0 & 0.01 & 0.47 \\
\hline & General health & 70.7 & $70.5 \pm 17.7$ & $70.5 \pm 14.6$ & $74.0 \pm 15.8$ & 0.4 & 0.59 & 0.04 \\
\hline
\end{tabular}

Data presented as mean \pm SD unless otherwise indicated. ${ }^{\star}$ Treatment effects maintained at follow-up; ${ }^{\dagger}$ Scores at pretest significantly different from normal controls; ' ${ }^{\dagger}$ cores at follow-up no longer significantly different from normal controls; ${ }^{\S}$ A higher score on the Short Form-36 Health Survey (SF-36) represents better physical functioning. $\eta^{2}$ Eta-squared; CIS-20R Checklist Individual Strength - Revised; IES Impact of Events Scale; SCL-90 Symptom Checklist 90; SIL Self-Inventory List

benefit at all from EMDR treatment; her pain intensity had not decreased during treatment or thereafter, and the pain had slightly increased 30 months after conclusion of treatment. This patient not only suffered from PLP, but also from hernia and CRPS before treatment and, meanwhile, her healthy foot was also affected by CRPS.

\section{DISCUSSION}

There is increasing evidence that painful conditions, such as PLP, may be the result of inappropriately stored or chronically activated pain memories $(9,10)$. In the present study, it was hypothesized that because EMDR is effective in treating traumatic memories, PLP (conceptualized as pain memory) might be effectively treated in a similar way. Our results indicate that focusing on unresolved trauma and pain-related memories might be a promising, noninvasive and relatively brief treatment strategy - at least for some patients.

Substantial reductions in chronic PLP were obtained. Of the eight participants who improved, four were considered completely pain free at three months follow-up. Almost three years after treatment, three of the six available participants were completely pain free. These findings are in line with previous reports on PLP treated with EMDR, in which $40 \%$

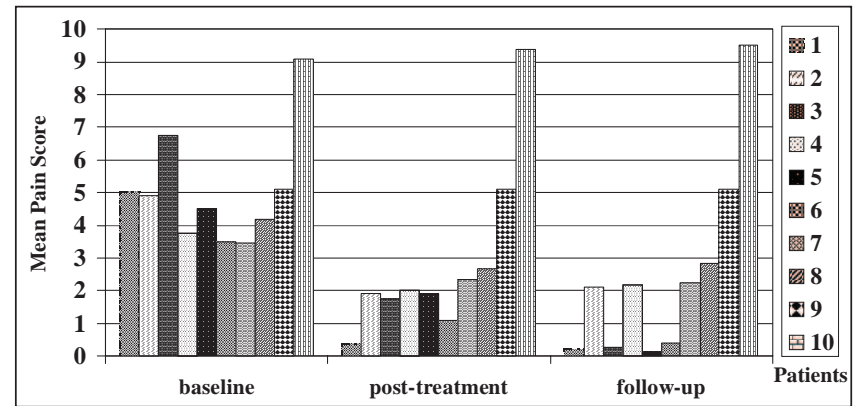

Figure 1) Mean pain scores (from the pain diary) per patient at baseline, post-treatment and at three months follow-up

(21) to $80 \%$ (23) of the participants showed almost complete remission. Furthermore, after treatment, the score on the bodily pain subscale (SF-36) fell within the normal range of the Dutch population. The mean total scores of the trauma scales decreased significantly over time and the only subscale that differed from the normal population at pretest normalized after treatment.

In the present study, the question arises as to why the treatment was effective for some, but not all participants. The first 
explanation could be that EMDR resolved the memories of events that triggered or maintained the present pain (19). More specifically, the EMDR standard protocol and the EMDR pain protocol used in the present study targeted the two types of somatosensory memories believed to be involved in PLP (ie, trauma-related pain flashbacks, and pain memories resulting from long-lasting or intense preamputation pain).

In this respect, two of our findings are important. First, albeit before treatment, only one participant fulfilled the criteria for PTSD, and the sample generally reported more trauma symptoms than the normal controls. In accordance herewith, $70 \%$ of the participants reported trauma- or pain-related memories that were affect laden and could subsequently be targeted. In three of the participants, no explicit disturbing trauma or pain-related memories could be identified. Despite the physical disability resulting from the amputation, these three participants regarded their operation and related events as positive because they considered the amputation to be a life-saving event. Interestingly, the two nonresponders belong to this group. Thus, the question arises as to whether it is necessary for participants to have access to an explicit amputation-related memory that is still affect laden to benefit from EMDR treatment.

Second, with regard to somatosensory memories due to preamputation pain, six participants reported preamputation pain and three of them had pain for longer than one year. The two nonresponders were among them. This raises the question of whether the chance of becoming pain free after EMDR treatment might be reduced for those who had preamputation pain of long duration. Katz and Melzack (9) suggest that the development of somatosensory memories may depend on a mechanism in which the threshold is sensitive to a combination of intensity and duration, so that intense pains of short duration (eg, gangrene), and long-lasting mild or innocuous sensations (eg, a bandage or wedding band) create sufficient excitation to produce long-term central changes. Therefore, future studies should include detailed information on the duration and intensity of the preamputation pain, in addition to characterization of the type of pain.

Another explanation for the effects of the present treatment is that EMDR modifies cortical plasticity by giving indirect sensory input (exposure) to the brain region that formerly represented the amputated limb. PLP and cortical reorganization are strongly correlated $(8,33)$, and cortical reorganization can be altered by changing the sensory input in the neural network either by strengthening it (34-36) or by taking it away $(37,38)$. This could also explain the promising results obtained in studies using noncustomary methods such as

\section{REFERENCES}

1. Jensen TS, Krebs B, Nielsen J, Rasmussen P. Immediate and long-term phantom limb pain in amputees: Incidence, clinical characteristics and relationship to pre-amputation limb pain. Pain 1985;21:267-78.

2. Nikolajsen L, Staehelin JT. Phantom limb pain. Curr Rev Pain 2000;4:166-70.

3. Horgan O, MacLachlan M. Psychosocial adjustment to lower-limb amputation: A review. Disabil Rehabil 2004;26:837-50.

4. Kooijman CM, Dijkstra PU, Geertzen JH, Elzinga A, van der Schans CP. Phantom pain and phantom sensations in upper limb amputees: An epidemiological study. Pain 2000;87:33-41. acupuncture (39), mirror therapy $(40,41)$, sensory discrimination training (34) and hypnosis (42). Concentrating on the phantom limb sensation or on phantom limb movements would activate brain areas corresponding with the original limb before amputation (43-45). Similarly, concentrating on the pain (as in the EMDR pain protocol) might activate the neural PLP network (46). Thus, another way in which trauma treatments similar to EMDR may affect PLP is through the induction of a functional reorganization (ie, restoration of the balance between excitatory and inhibitory input) in brain circuitry involved in PLP. If the effect of EMDR on PLP is the result of indirect sensory input, then EMDR should also be able to alter or reverse cortical reorganization. Future studies using brain imaging techniques may elucidate this process.

One limitation of the present study is the small number of participants. In addition, due to the absence of a (placebo) control group, the reported improvements could be due to the passage of time rather than to the specific intervention. Finally, although the effect sizes (based on intention-to-treat analysis) were large enough to suggest a real effect, and in most cases pain reduction was maintained at long-term follow-up, it is possible that nonspecific factors (such as attention, hope and support from the therapist) contributed to reduction in pain intensity. However, this seems unlikely because our participants had severe disabling pain for many years (mean 13.6 years) and had not responded to previous pharmacological, psychological and noncustomary treatments.

The results of our study support the idea that treatment aimed at processing unresolved trauma and pain-related memories can reduce the intensity of PLP by reducing the affective dimension of these memories or by integrating the somatic memory components (47). For this approach to be effective, it seems that subjects need access to amputation-related memories, which are still disturbing to them at the time of the treatment, and/or they should not have long-lasting preamputation pain. Although the present results are promising, besides the need for randomized controlled trials, multiple replications with single subjects, time series analyses or multiple baseline approaches are warranted because these approaches may contribute to establishing the efficacy of trauma-focused approaches for PLP and may help to elucidate the processes involved.

ACKNOWLEDGEMENTS: The authors thank all the participants for their contributions to this study, as well as B Tinker and $\mathrm{S}$ Wilson for sharing their experiences with EMDR and PLP. They also thank M Brouwer, M Mutsaers and J Kools for their contribution to the data collection. The present pilot study did not receive any funding. The authors have no conflicts of interest to declare.
5. Halbert J, Crotty M, Cameron ID. Evidence for the optimal management of acute and chronic phantom pain: A systematic review. Clin J Pain 2002;18:84-92.

6. Nikolajsen L, Jensen TS. Phantom limb pain. Br J Anaesth 2001;87:107-16.

7. Flor H. Phantom-limb pain: Characteristics, causes, and treatment. Lancet Neurol 2002;1:182-9.

8. Flor H. Cortical reorganisation and chronic pain: Implications for rehabilitation. J Rehabil Med 2003;(41 Suppl):66-72.

9. Katz J, Melzack R. Pain 'memories' in phantom limbs: Review and clinical observations. Pain 1990;43:319-36. 
10. Flor H. Painful memories. Can we train chronic pain patients to 'forget' their pain? EMBO Rep 2002;3:288-91.

11. Salomons TV, Osterman JE, Gagliese L, Katz J. Pain flashbacks in posttraumatic stress disorder. Clin J Pain 2004;20:83-7.

12. Schreiber S, Galai-Gat T. Uncontrolled pain following physical injury as the core-trauma in post-traumatic stress disorder. Pain 1993;54:107-10.

13. Whalley MG, Farmer E, Brewin CR. Pain flashbacks following the July 7th 2005 London bombings. Pain 2007;132:332-6.

14. Melzack R. Pain and the neuromatrix in the brain. J Dent Educ 2001;65:1378-82.

15. Flor H, Nikolajsen L, Staehelin JT. Phantom limb pain: A case of maladaptive CNS plasticity? Nat Rev Neurosci 2006;7:873-81.

16. Ray AL, Zbik A. Cognitive behavioral therapies and beyond. In: Tollison CD, Satterhwaite JR, Tollsion JW, eds. Practical Pain Management, 3rd edn. Philadelphia: Lippincott, 2001:189-209.

17. Schneider J, Hofmann A, Rost C, Shapiro F. EMDR and phantom limb pain, theoretical implications, case study, and treatment guidelines. J EMDR Pract Res 2007;1:45.

18. Bisson JI, Ehlers A, Matthews R, Pilling S, Richards D, Turner S. Psychological treatments for chronic post-traumatic stress disorder. Systematic review and meta-analysis. Br J Psychiatry 2007;190:97-104

19. Shapiro F. Eye Movement Desensitization and Reprocessing (EMDR): Basic Principles, Protocols, and Procedures, 2nd edn. New York: The Guilford Press, 2001.

20. Russell MC. Treating traumatic amputation-related phantom limb pain: A case study utilizing eye movement desensitization and reprocessing within the armed services. Clin Case Studies 2008;7:136-53.

21. Schneider J, Hofmann A, Rost C, Shapiro F. EMDR in the treatment of chronic phantom limb pain. Pain Med 2008;9:76-82.

22. Tinker RH, Wilson SA. The Phantom Limb Pain Protocol. EMDR Solutions: Pathways to Healing. New York: Norton WW, 2005:147-59.

23. Wilensky M. Eye movement desensitization and reprocessing (EMDR) as a treatment for phantom limb pain. J Brief Ther 2006;5:31-44.

24. van Vliet IM, Leroy H, van Megen HJGM. M.I.N.I. Internationaal Neuropsychiatrisch Interview; Nederlandse versie 5.0.0.-R. 2000.

25. Arrindell WA, Ettema JHM. SCL-90 Handleiding bij een multidimensionele psychopathologie-indicator. Lisse: Swets \& Zeitlinger, 1975.

26. Vercoulen JHMM, Alberts M, Bleijenberg G. De Checklist Individual Strength (CIS). Gedragstherapie 1999;32:131-6.

27. van der Ploeg E, Mooren TT, Kleber RJ, van der Velden PG Brom D. Construct validation of the Dutch version of the impact of event scale. Psychol Assess 2004;16:16-26.

28. Hovens JE, Bramsen I, van der Ploeg HM. Self-rating inventory for posttraumatic stress disorder: Review of the psychometric properties of a new brief Dutch screening instrument. Percept Mot Skills 2002;94:996-1008.

29. Aaronson NK, Muller M, Cohen PD, et al. Translation, validation, and norming of the Dutch language version of the SF-36 Health
Survey in community and chronic disease populations. J Clin Epidemiol 1998;51:1055-68.

30. de Roos C, Veenstra AC. EMDR pain protocol for current pain. In: Luber M, ed. Eye Movement Desensitization and Reprocessing (EMDR): Special Populations. New York: Springer, 2009.

31. Grant M. Pain control with EMDR. Denver: Mentor Books, 1999.

32. Servan-Schreiber D, Schooler J, Dew MA, Carter C, Bartone P. Eye movement desensitization and reprocessing for posttraumatic stress disorder: A pilot blinded, randomized study of stimulation type. Psychother Psychosom 2006;75:290-7.

33. Flor H, Elbert T, Knecht S, et al. Phantom-limb pain as a perceptual correlate of cortical reorganization following arm amputation. Nature 1995;375:482-4.

34. Flor H, Denke C, Schaefer M, Grusser S. Effect of sensory discrimination training on cortical reorganisation and phantom limb pain. Lancet 2001;357:1763-4.

35. Flor $\mathrm{H}$. The modification of cortical reorganization and chronic pain by sensory feedback. Appl Psychophysiol Biofeedback 2002;27:215-27.

36. Lotze M, Grodd W, Birbaumer N, Erb M, Huse E, Flor H. Does use of a myoelectric prosthesis prevent cortical reorganization and phantom limb pain? Nat Neurosci 1999;2:501-2.

37. Birbaumer N, Lutzenberger W, Montoya P, et al. Effects of regional anesthesia on phantom limb pain are mirrored in changes in cortical reorganization. J Neurosci 1997;17:5503-8.

38. Huse E, Larbig W, Flor H, Birbaumer N. The effect of opioids on phantom limb pain and cortical reorganization. Pain 2001;90:47-55.

39. Bradbrook D. Acupuncture treatment of phantom limb pain and phantom limb sensation in amputees. Acupunct Med 2004;22:93-7.

40. MacLachlan M, Desmond D, Horgan O. Psychological correlates of illusory body experiences. J Rehabil Res Dev 2003;40:59-65.

41. Ramachandran VS, Rogers-Ramachandran D, Cobb S. Touching the phantom limb. Nature 1995;377:489-90.

42. Oakley DA, Whitman LG, Halligan PW. Hypnotic imagery as a treatment for phantom limb pain: Two case reports and a review. Clin Rehabil 2002;16:368-77.

43. Lotze M, Montoya P, Erb M, et al. Activation of cortical and cerebellar motor areas during executed and imagined hand movements: An fMRI study. J Cogn Neurosci 1999;11:491-501.

44. Lotze M, Flor H, Grodd W, Larbig W, Birbaumer N. Phantom movements and pain. An fMRI study in upper limb amputees. Brain 2001;124:2268-77.

45. Rosen G, Hugdahl K, Ersland L, et al. Different brain areas activated during imagery of painful and non-painful 'finger movements' in a subject with an amputated arm. Neurocase 2001;7:255-60.

46. Willoch F, Rosen G, Tolle TR, et al. Phantom limb pain in the human brain: Unraveling neural circuitries of phantom limb sensations using positron emission tomography. Ann Neurol 2000;48:842-9

47. Maxfield L. Current status and future directions for EMDR research. J EMDR Pract Res 2007;1:6-14. 


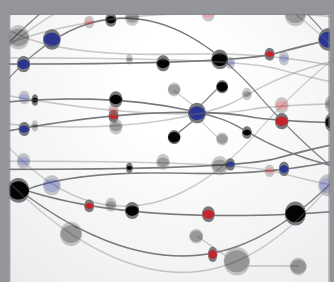

The Scientific World Journal
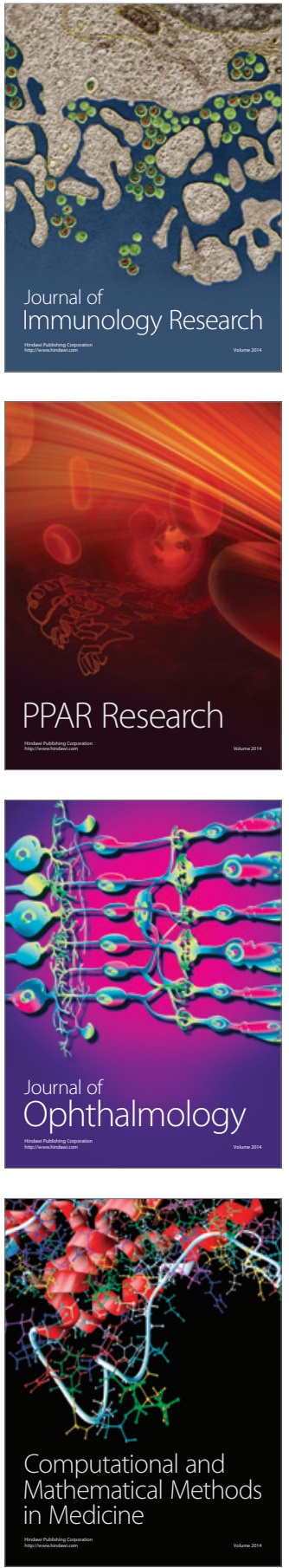

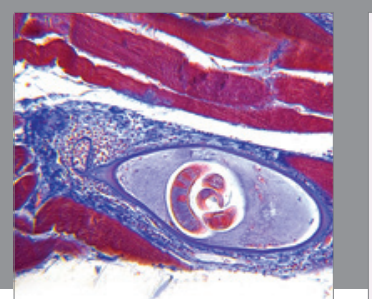

Gastroenterology Research and Practice

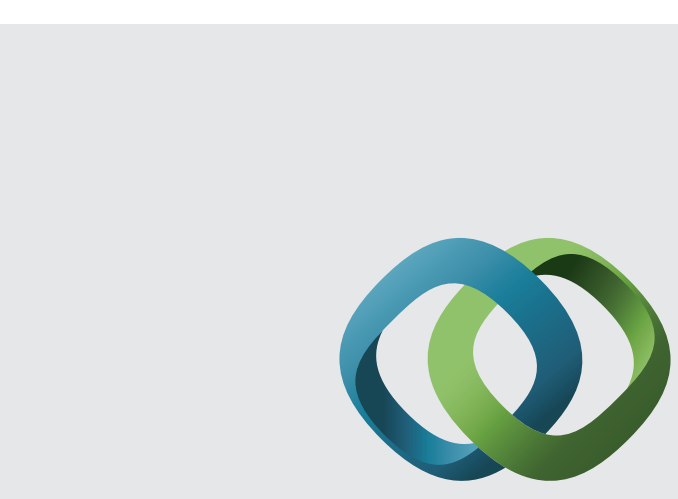

\section{Hindawi}

Submit your manuscripts at

http://www.hindawi.com
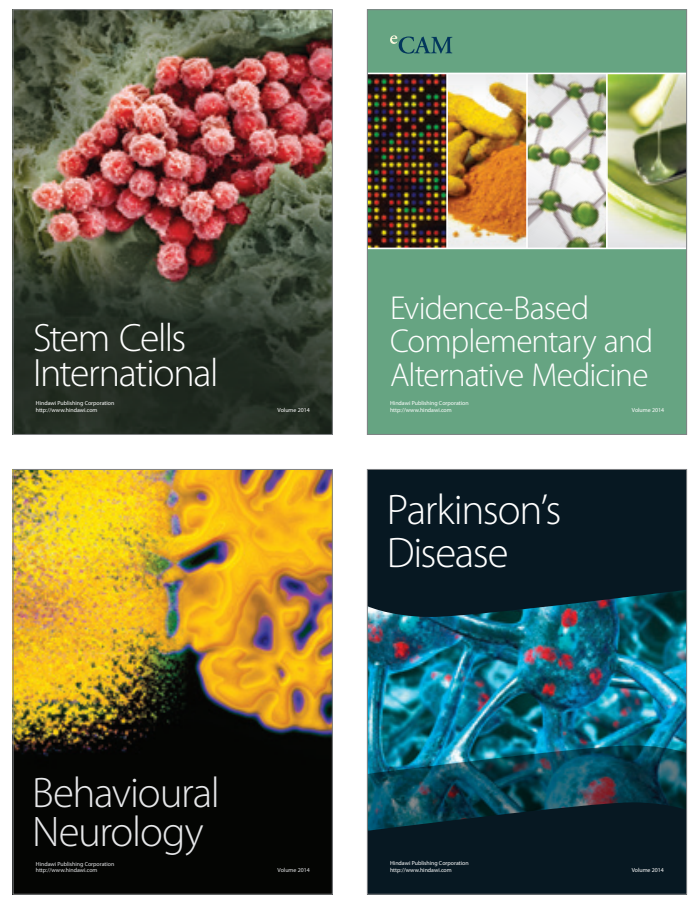
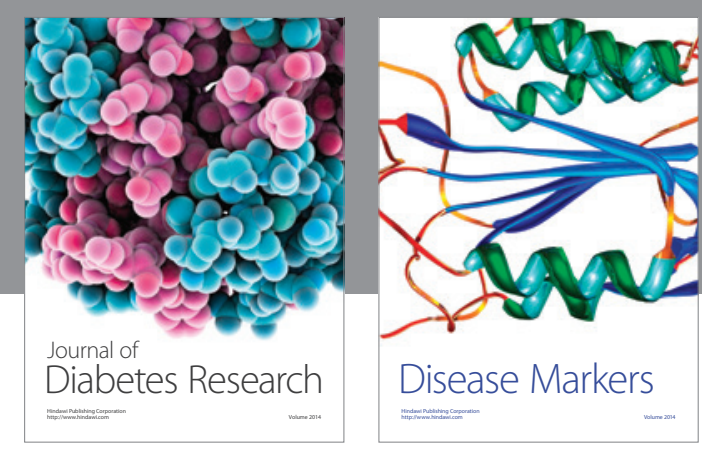

Disease Markers
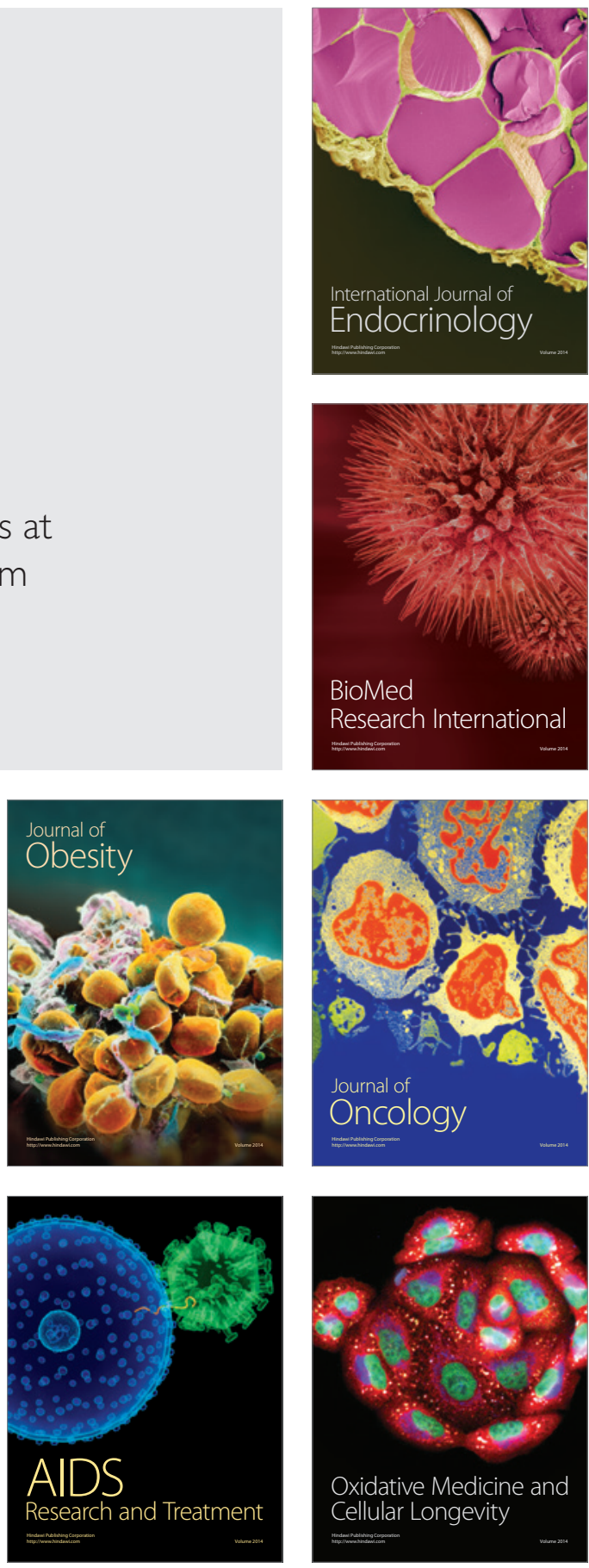